\title{
Calcium-sensing receptor is involved in the pathogenesis of fat emulsion-induced insulin resistance in rats
}

\author{
JIAMING XIE ${ }^{1,2^{*}}$, YUAN JIANG ${ }^{1 *}$, YUNA KAN $^{1}$, JING ZHAO $^{1}$, HAIXUE KUANG $^{1,3}$ and PENGLING GE ${ }^{1}$ \\ ${ }^{1}$ Department of Pharmacology, School of Basic Medical Sciences, Heilongjiang University of Chinese Medicine, Harbin, \\ Heilongjiang 150040; ${ }^{2}$ The Key Laboratory of Myocardial Ischemia, Harbin Medical University, Ministry of Education, \\ Harbin, Heilongjiang $150086 ;{ }^{3}$ The Key Laboratory of Chinese Ministry of Education, Department of Traditional Chinese Medicine, \\ School of Pharmacology, Heilongjiang University of Chinese Medicine, Harbin, Heilongjiang 150040, P.R. China
}

Received August 28, 2014; Accepted March 20, 2015

DOI: $10.3892 / \mathrm{mmr} .2015 .3644$

\begin{abstract}
A high-fat diet not only leads to obesity, but also leads to a predisposition towards insulin resistance (IR), which is characterized by hyperinsulinemia and reduced glucose tolerance. However, the etiology of IR remains to be fully elucidated. The present study investigated whether calcium-sensing receptor (CaSR) is involved in the development of IR in rats fed a high-fat diet. IR was induced in the rats by feeding with a fat emulsion via gavage for 2, 4, 6 or 8 weeks. Reverse transcription-quantitative polymerase chain reaction (RT-q-PCR) and western blot analysis were performed to investigate whether CaSR-associated proteins were affected. The gavage of fat emulsion for 8 weeks induced a notable decline in the insulin sensitivity index (ISI) between -4.98 and -5.60 . With 6 weeks of gavage, a significant difference in the ISI was observed between the IR and control groups. The results of the RT-qPCR and western blot analysis demonstrated that phosphatidylinositol 3-kinase/Akt pathway, which is a pathway closely associated with the CaSR signaling pathway, was significantly inhibited in the rats with IR. The results of the present study provided evidence that CaSR is associated with the development of IR in rats fed a high-fat diet and suggested that CaSR may be important in the pathogenesis of diabetes.
\end{abstract}

\section{Introduction}

The number of individuals with a high-fat diet and sedentary lifestyle have increased worldwide. A high-fat diet causes

Correspondence to: Professor Haixue Kuang or Dr Pengling Ge, Department of Pharmacology, School of Basic Medical Sciences, Heilongjiang University of Chinese Medicine, 24 Heping Road, Harbin, Heilongjiang 150040, P.R. China

E-mail:khxoffice@hljucm.net

E-mail: penglingge@126.com

*Contributed equally

Key words: high-fat diet, calcium-sensing receptor, insulin resistance, diabetes severe damage to organs and leads to serious complications (1). The excessive intake of calories in the diet and lack of exercise lead to a predisposition towards obesity (2). Obesity is a key risk factor for a decrease in insulin sensitivity, also referred to as IR (3), in which the sensitivities of organs or tissues to insulin are compromised and can be lost, and the uptake and utilization efficiency of blood glucose are decreased (4). IR may trigger type 2 diabetes, metabolic syndrome, uarthritis or cardiovascular diseases (1). However, the mechanism of IR remain to be fully elucidated. A previous study indicated that the phosphatidylinositol 3-kinase (PI3K)/Akt signaling pathway, particularly the activation of Akt, may be important in the development of IR (5). However, the exact role of these proteins in the pathogenesis of IR remains to be elucidated. Calcium-sensing receptor (CaSR) is an integral membrane protein, which belongs to the $\mathrm{G}$ protein-coupled receptor 3 family (6). CaSR is expressed predominantly in the liver, but is also expressed in the muscle, placenta and brain. CaSR has been observed to be closely associated with the PI3K/Akt pathway in cardiovascular and bone systems $(7,8)$. Another study reported that a CaSR antagonist inhibited Akt activity, while its agonist significantly enhanced the activation of Akt (9). Therefore, CaSR may be involved in the pathophysiology of IR by affecting Akt. In the present study, the possible correlation between the expression of CaSR and the fat emulsion-induction of IR in rats was investigated.

\section{Materials and methods}

Fat emulsion preparation. A $100 \mathrm{ml}$ fat emulsion, containing lard (20\%; Lihongde Co., Ltd., Tianjin, China), propylthiouracil (1\%; Sigma-Aldrich, St. Louis, MO, USA), cholesterol $(5 \%)$, sodium glutamate $(1 \%)$, fructose $(5 \%)$ (Shanghai Huishi Biochemical Co. Ltd., Shanghai, China), sucrose (5\%; Tianjin Basifu Chemical Co., Ltd., Tianjin China) and edible salt (6\%; China National Salt Industry Co., Ltd., Beijing, China) in Tween 80 (20\%; Tianjin Jin Feng Chemical Co., Ltd., Tianjin, China) and propylene glycol (30\%; Tianjin Kemiou Chemical Reagent Co., Ltd., Tianjin China), was prepared by adding $7 \mathrm{ml}$ distilled water to $100 \mathrm{ml}$, as previously reported (4), and stored at $4^{\circ} \mathrm{C}$ until used to feed the animals. The procedures involving the use of animals were approved by the Animal 
Experimental Ethical Committee of Heilongjiang University of Chinese Medicine (Harbin, China).

Animals and rat IR model. Adult, 2-month-old male Wistar rats were purchased from Yisi Lab Animal Technology Co. (Changchun, China), and were housed individually in an air-conditioned facility with a 12 -h light/dark cycle at $25 \pm 1^{\circ} \mathrm{C}$ and $50 \pm 5 \%$ humidity, and supplied with food and water ad libitum. A total of 40 Wistar rats were randomly divided into a control group $(n=20)$ and an IR model group $(n=20)$. The rats in the control group were further divided into four subgroups: 2 week control $(n=5), 4$ week control $(n=5), 6$ week control $(n=5)$ and 8 week control $(n=5)$. Accordingly, the rats in the IR model group were divided into four corresponding subgroups with the same durations and numbers of animals as the control groups. The rats in the IR model group were administered with a fat emulsion $(10 \mathrm{ml} / \mathrm{kg})$ via gavage every day for 2, 4, 6 or 8 weeks, whereas the control rats were administered with the same quantity of distilled water, at the same time-points. Following $12 \mathrm{~h}$ fasting, blood samples $(1.5 \mathrm{ml})$ were collected from the abdominal aorta of the rats to examine the concentrations of insulin and blood glucose. Glucose meter determination (Accu-Chek; Roche, Dublin, Ireland) was used to measure the levels of fasting blood glucose (FBG), according to the manufacturer's instruction. An enzyme-linked immunosorbent assay (ELISA) kit (R\&D Systems, Inc., Minneapolis, MN, USA) was used to investigate the levels of fasting insulin (FINS). The rat insulin sensitivity index (ISI) was calculated using the following equation: ISI $=\ln [1 /($ FBG $x$ FINS) $]$. At the end of the experiments, the animals were sacrificed by cervical dislocation under 1\% pentobarbital (Sigma-Aldrich) and liver and skeletal muscle samples were harvested from all animals and maintained in liquid nitrogen.

Immunofluorescence. The fresh liver and skeletal muscle tissues were embedded in Optimum Cutting Temperature compound (Thermo Fisher Scientific, Waltham, MA, USA). A freezing microtome (Thermo Fisher Scientific) was used to prepare frozen sections of skeletal muscle and liver. The frozen tissue sections were then fixed with cold acetone (Luoyang Haohua Chemical Reagent Co., Ltd., Henan, China) for $10 \mathrm{~min}$. Following rinsing with phosphate-buffered saline (PBS; GE Healthcare Life Sciences, Beijing, China), blocking with $0.1 \%$ Triton X-100 (Solarbio, Beijing, China) and $10 \%$ bovine serum albumin (Thermo Fisher Scientific) for $30 \mathrm{~min}$ at room temperature, the sections were incubated with rabbit polyclonal CaSR antibody (1:1,000; ab137408; Abcam, MA) at room temperature for another $1 \mathrm{~h}$. Following three 10 min washes with PBS, the sections were then incubated with fluorescein isothiocyanate (FITC)-labeled second antibody at room temperature for a further $1 \mathrm{~h}$. Subsequently, 4,6-diamidino-2-phenylindole (Solarbio) was used to label the cell nuclei, and the sections were then washed, mounted and examined using a fluorescence microscope (IX51; Olympus, Tokyo, Japan).

RNA isolation. The liver and skeletal muscle mRNA was extracted using a TRIzol kit (Invitrogen Life Technologies, Carlsbad, CA, USA), according to the manufacturer's instructions. Subsequently, the total mRNA from all the samples was quantified using an ultraviolet and visible spectrophotometer (BioSpec-nano; Shimadzu Corporation, Kyoto, Japan).

Reverse transcription-quantitative polymerase chain reaction $(R T-q P C R)$. The total mRNAs from liver and skeletal muscle were reversely transcribed to cDNA using AccuPower RocketScript RT Premix (Bioneer Corporation, Daejeon, Korea). qPCR was then performed in duplicate using an AccuPower GreenStar qPCR master mix (Bioneer Corporation), according to the manufacturer's instructions. CaSR primers (forward 5'-ttctttgaacetggacgacgagt-3' and reverse 3'-gcgaggaaggatttgtac-5') were used for amplifying the fragments. qPCR was performed using a q-PCR device from Bio-Rad Laboratories (Hercules, CA, USA) using the following steps: $95^{\circ} \mathrm{C}$ for $10 \mathrm{~min}$, followed by 40 two-step cycles of $95^{\circ} \mathrm{C}$ for $15 \mathrm{sec}$ and $47^{\circ} \mathrm{C}$ for $1 \mathrm{~min}$. $\beta$-actin (forward primer $5^{\prime}$-gtcaggtcatcactatcggcaat-3' and reverse 3'-agaggtctttacggatgtcaacgt-5') was used as an internal control, The relative expression values were calculated using the $2^{-\Delta \Delta C t}$ method. The qPCR products also were visualized via agarose gel electrophoresis (AGE) to identify non-specific bands.

Western blot analysis. Protein samples $(50 \mu \mathrm{g})$ were separated on a $10 \%$ SDS gel (Beyotime Institute of Biotechnology, Jiangsu, China) and transferred onto a polyvinylidene (PVDF; Invitrogen Life Technologies) membrane. The nonspecific proteins were blocked using $5 \%$ non-fat dry milk at $37^{\circ} \mathrm{C}$ for $1 \mathrm{~h}$. Subsequently, each PVDF membrane was incubated with the following primary antibodies at $4^{\circ} \mathrm{C}$ for $12 \mathrm{~h}$ : Rabbit polyclonal anti-CaSR (1:1,000; ab137408; Abcam, Cambridge, MA, USA); rabbit polyclonal protein kinase B (1:1,000; ab106693; Abcam), rabbit monoclonal phosphorylated (phospho)-Akt at Ser473 (1:1,000; ab81283; Abcam) and rabbit monoclonal phospho-Akt at T308 (1:1,000; Cell Signaling Technology, Inc. Danvers, MA, USA). The membranes were then incubated for $2 \mathrm{~h}$ with the goat anti-rabbit horseradish peroxidase-conjugated affiniPure secondary IgG antibody (1:1,000; ab6721; Abcam) and then visualized using chemiluminescence (Solarbio).

Statistical analysis. The results are presented as the mean \pm standard error of the mean (SEM), and the data were analyzed with SPSS, version 19.0 (IBM SPSS, Armonk, NY, USA). The data were analyzed using Student's t-tests $\mathrm{P}<0.05$ was considered to indicate a statistically significant difference.

\section{Results}

Body weight, FBG, FINS and ISI in rats with fat emulsion-induced $I R$. The results demonstrated that a high-fat diet induced a significantly increased body weight in the rats with IR, and there were significant differences between the group fed for 6 weeks and that fed for 8 weeks (Fig. 1A). The ISI value decreased between -4.98 and -5.60 following 8 weeks on a high fat diet in the rats with IR, and there was a significant difference in the ISI between the group fed for 6 weeks and that fed for 8 weeks (Fig.1B). In addition, as shown in Fig. 1C, the FBG values in the rats with IR were markedly higher following 4, 6 and 8 weeks high-fat gavage-feeding compared with those in the control group. Following 8 weeks on the high-fat diet, the FINS was significantly increased in the IR group. 

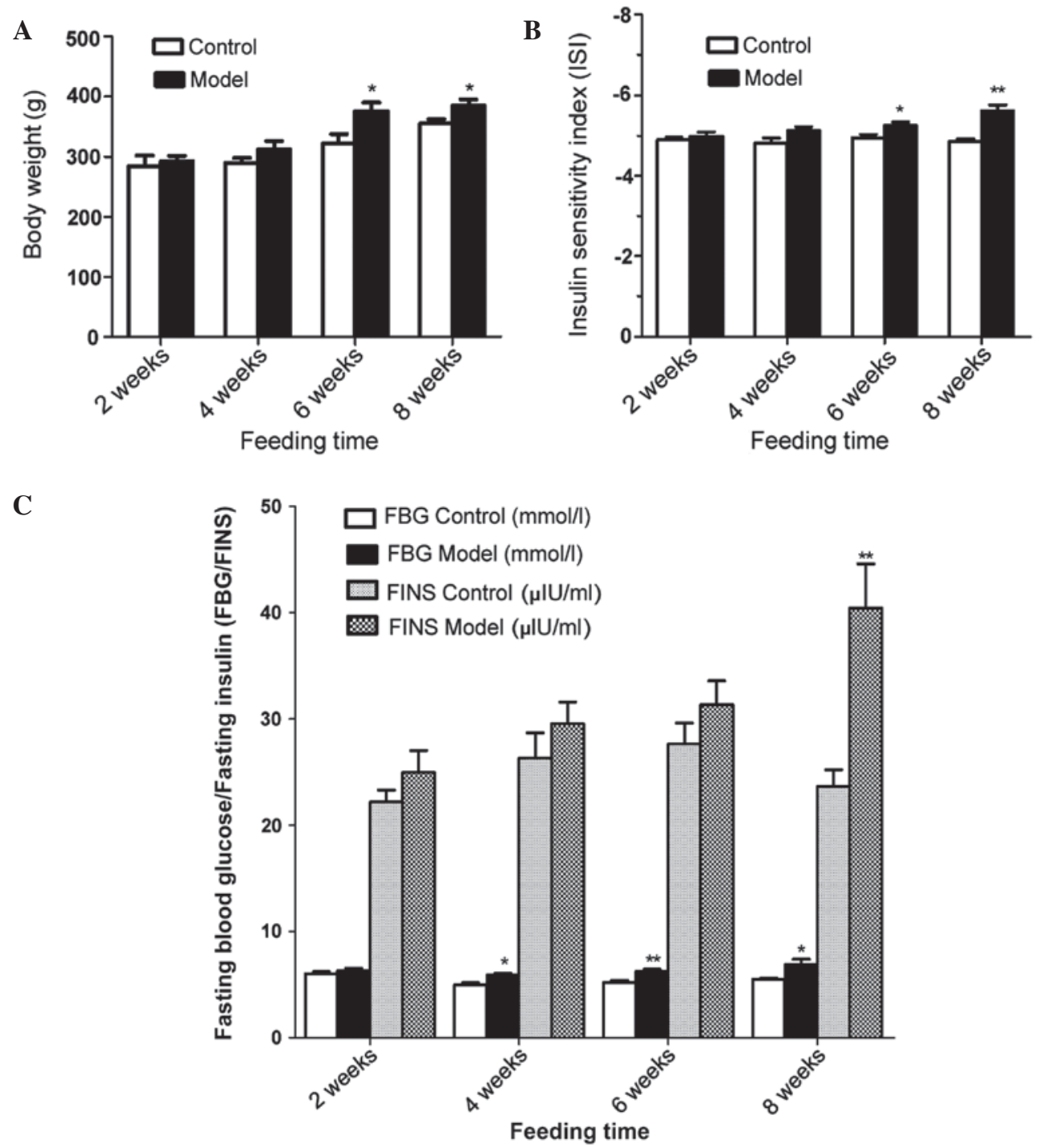

Figure 1. Body weights, ISI, FBG and FINS in the control and IR groups following 2, 4, 6 and 8 weeks feeding. (A) Weights of rats in the IR model group were greater than those in the control group. After 6 weeks, the weights of the rats in the IR model group were significantly higher than those in the control group ( $\mathrm{n}=10$; $\mathrm{P}<0.05)$. (B) Significant increases in ISI were observed among the control and IR groups after 6 weeks $(n=10 ; \mathrm{P}<0.05)$ and 8 weeks $(\mathrm{n}=5$; $\mathrm{P}<0.01)$. $(\mathrm{C})$ Significant increases in FBG were observed in the IR group after 4 weeks $(n=15 ; P<0.05), 6$ weeks $(n=10 ; P<0.01)$ and 8 weeks $(n=5 ; P<0.05)$ compared with their respective control groups. Significant increases in FINS were observed in the IR group after 8 weeks compared with the control group $(\mathrm{n}=5$; $\mathrm{P}<0.01)$.. All data are presented as the mean \pm standard error of the mean. ${ }^{*} \mathrm{P}<0.05 ;{ }^{* *} \mathrm{P}<0.01$. IR, insulin resistance; ISI, insulin sensitivity index; FBG; fasting blood glucose; FINS; fasting insulin.

Protein expression of CaSR in the liver and skeletal muscle of rats. To confirm the expression of CaSR in the rat liver and skeletal muscle, immunostaining was performed on the liver and skeletal muscle sections of rats in the control and IR groups (2 weeks). As shown in Fig. 2, the liver and skeletal muscle demonstrated positive staining for the expression of CaSR.

Expression of CaSR and activity of Akt in the skeletal muscle and liver tissues of rats with or without a high-fat diet. The present study subsequently examined whether CaSR was regulated in response to a high-fat diet in rats. The mRNA expression levels of CaSR in the liver and skeletal muscles were downregulated in the IR group compared with the control group (Fig. 3). The mRNA expression of CaSR was significantly reduced in the IR group following 2, 4, 6 and 8 weeks a high-fat diet. In the muscle tissues, although no significant difference in the mRNA expression levels of CaSR were observed between the two groups following 2 weeks of feeding, the mRNA level of CaSR was significantly reduced following 4, 6 and 8 weeks of feeding.

The protein expression levels of CaSR in the two groups were also investigated using western blot analysis (Fig. 4). A notable decrease in the protein expression of CaSR was observed in the IR group, compared with the control group. The present study then assessed whether there was an association between the protein expression of CaSR and Akt activity. As shown in Fig. 4, the expression levels of phospho-Akt (Ser473 and Thr308) in the liver and skeletal muscles were also significantly lower in the IR group than in the control group following 6 and 8 weeks of a high-fat diet.

\section{Discussion}

The aim of the present study was to understand the molecular mechanism responsible for high-fat diet-induced IR in a rat model. At present, several methods are used to establish an IR 


\section{2 week control group}

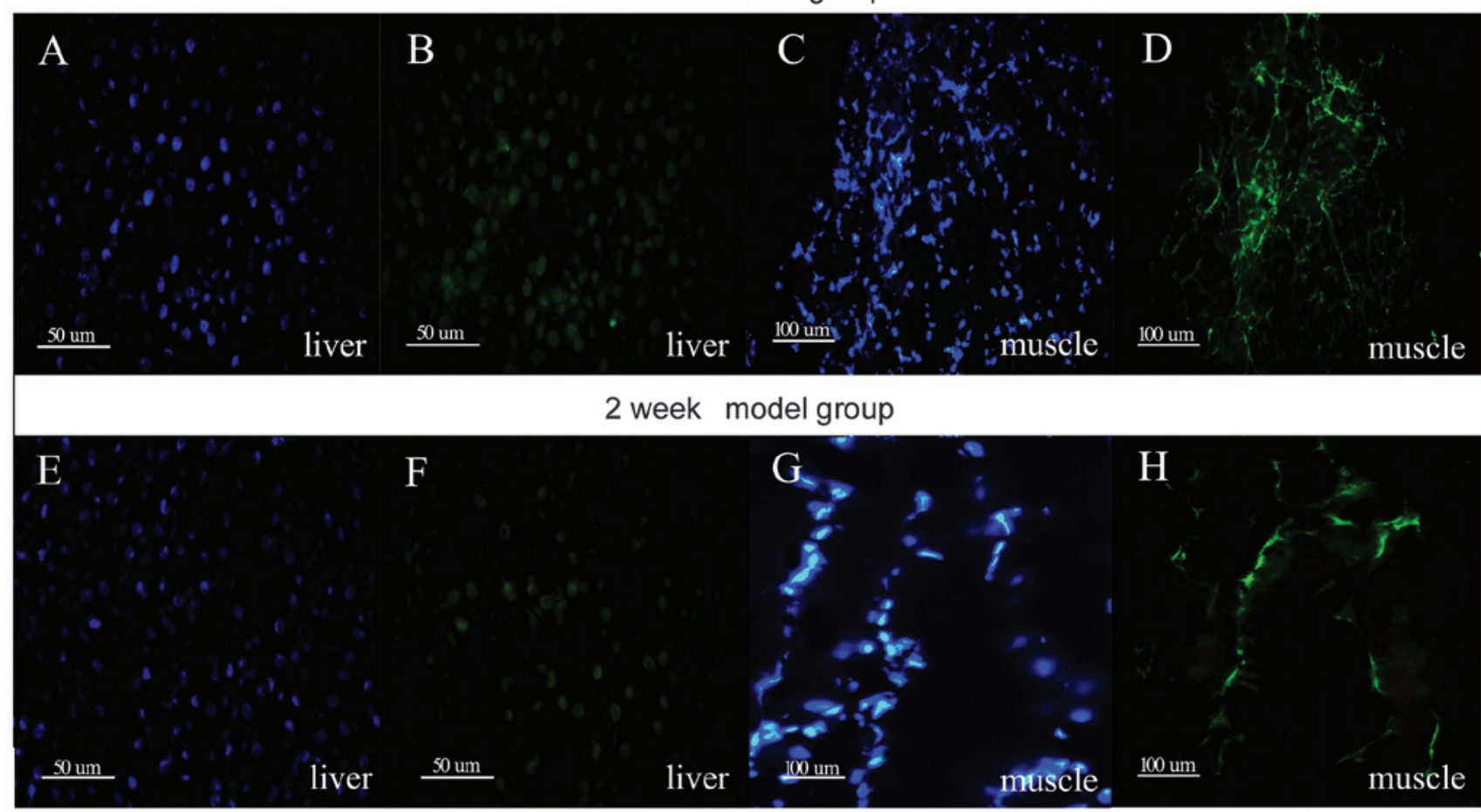

Figure 2. Immunofluorescence images of the protein expression levels in the rat liver and skeletal muscle sections from the (A-D) control and (E-H) insulin resistant rats. Staining for (A, C, E and G) 4,6-diamidino-2-phenylindole (blue) and (B, D, F and H) calcium-sensing receptor (green) was performed. Staining results demonstrate that compared with the control group, the calcium-sensing receptor protein (green) in the model group was significantly reduced.

A

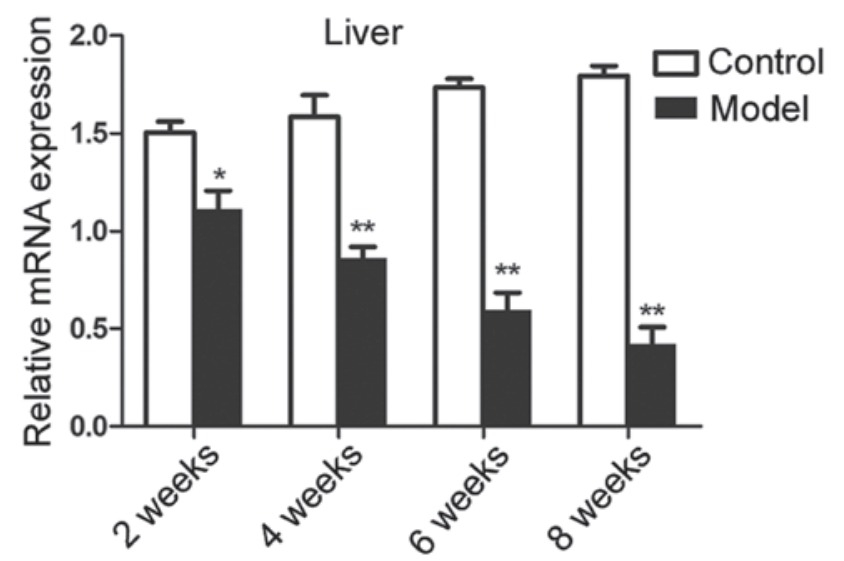

Feeding time
B

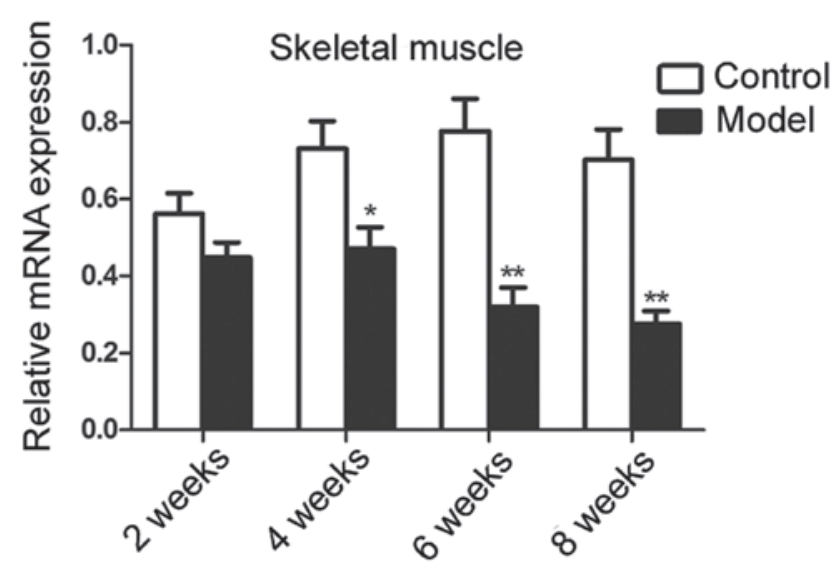

Feeding time

Figure 3. Gene expression levels of calcium-sensing receptor in the (A) liver and (B) skeletal muscle in the control group ( $\mathrm{n}=5$ ) and insulin resistant group ( $\mathrm{n}=5$ ). All data are presented as the mean \pm standard error of the mean. ${ }^{*} \mathrm{P}<0.05$ and ${ }^{* *} \mathrm{P}<0.01$, vs. control group.

model, including genetic modification and feeding a high-fat diet (10). In the present study, an IR model was established by feeding rats with a fat emulsion via gavage, which enabled control of the daily diet. The rats in the control group were fed distilled water by gavage, in order to mimic possible damage to the esophagus, which may occur due to gavage feeding in the fat emulsion group. Using this animal model, the present study aimed to clarify whether CaSR was involved in the pathogenesis of fat emulsion-induced IR. The results demonstrated that feeding rats fat emulsion for 6 weeks resulted in significant differences in the ISI, FINS, FBG and body weights between the control and IR groups. In addition, the high-fat diet had time-dependent effects on IR in the rats fed a high fat diet.

IR refers to the loss of the sensitivity of body target organs or tissues to insulin and the failure of insulin to promote glucose uptake (3). The liver and skeletal muscles are important in bodily glucose uptake (11) and are considered to be the major target tissues of insulin $(12,13)$. A previous study reported that CaSR knockdown compromised receptor-induced insulin secretion in a genetically modified animal model (14). Therefore, CaSR 

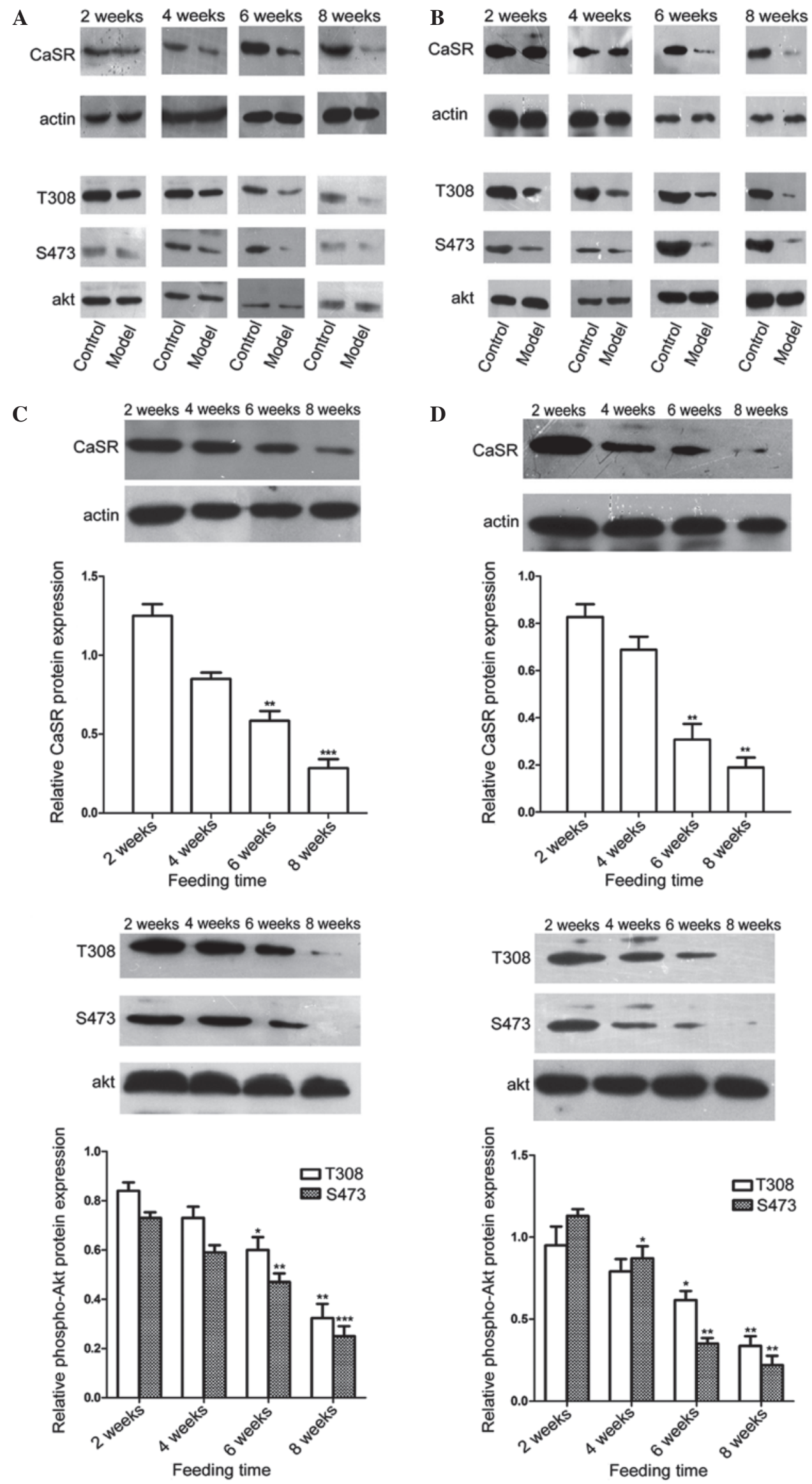

Figure 4. Protein expression of CaSR and phospho-Akt (Thr308 and Ser473) in the (A) liver and (B) skeletal muscle of the rats in the IR and control groups. CaSR and phospho-Akt (Thr308 and Ser473) in the IR model groups $(n=5)$ following different durations of treatment $(2,4,6$ and 8 weeks) in the (C) liver and (D) skeletal muscle. All data are presented as the mean \pm standard error of the mean. ${ }^{*} \mathrm{P}<0.05,{ }^{* *} \mathrm{P}<0.01$ and ${ }^{* * *} \mathrm{P}<0.001$, vs. IR group at 2 weeks. CaSR, calcium-sensing receptor; IR, insulin resistance; phospho, phosphorylated. 
may be a potential factor in increasing glucose-induced insulin secretion. Homozygous genetic mutations and mitochondrial DNA mutations of CaSR can lead to episodes of type 2 diabetes (15). All these previous findings suggest that the CaSR may be involved in IR. However, the exact role of CaSR in these processes remains to be fully elucidated.

In the present study, the expression levels of CaSR were examined in the skeletal muscle and liver tissues of rats using immunofluorescent staining, which provided a foundation for further investigation. Using RT-qPCR, marked decreases in the gene expression of CaSR were observed in the liver and skeletal muscle tissues of rats with high-fat diet-induced IR, which was negatively correlated with IR severity in these animals.

Several studies have demonstrated that the PI3K/Akt pathway is a key factor during the pathological changes of IR, particularly the activity of certain downstream kinases of Akt, including protein kinase B and Rac (5). It has been established that the mechanism by which growth factors and insulin increase Akt activity involve the phosphorylation of the Thr308 and Ser473 sites of Akt (5). Phosphorylation at these two sites is an important step for the function of Akt in survival and glycogenesis (16-18). Akt is also involved in cell cycle regulation and may be involved in the regulation of insulin in glucose transport $(19,20)$. In the liver and muscles, insulin induces the phosphorylation of Akt at Thr308 and Ser473 via the activation of PDK1 (21). In the present study, immunofluorescence staining revealed that the protein expression of CaSR was decreased in the IR group following 2 weeks on a high-fat diet. The decreased expression of CaSR was confirmed using western blot analysis. In addition, a significant decrease was observed in the phosphorylation of Akt in the IR group following 2, 4, 6 and 8 weeks of high-fat diet feeding. These results suggested that the PI3K/Akt pathway was inhibited following feeding with the high-fat diet. A significant reduction was also observed in the protein expression of CaSR in the IR group, with a notable increase in the levels of IR in these animals. These data suggested that CaSR may have been responsible for the development of IR in this high-fat animal model by inhibiting the activity of Akt in the PI3k/Akt pathway.

In conclusion, the present study demonstrated the expression of CaSR in the liver and skeletal muscles in rats. A fat emulsion diet caused a significant decrease in the expression of CaSR in the IR rat model. The data also suggested that the expression of CaSR was closely associated with the activity of the PI3K/ Akt pathway, which is an important signaling pathway leading to IR. These findings may offer a novel area of investigation of the underlying molecular mechanisms by which IR occurs in animals fed a high-fat diet.

\section{Acknowledgements}

The authors would like to thank Dr Shaohong Fang from the Key Laboratory of Myocardial Ischemia, Harbin Medical University of the Chinese Ministry of Education for her assistance in technical support. This study was supported by grants from the National Natural Science Foundation of China (no. 81273650), Chinese Ministry of Science and Technology (no. 2012ZX09103201-018), Natural Science Foundation of Heilongjiang province (no. LC2011C03); Harbin
Science and Technology Bureau of Heilongjiang Province (no. 2011RFLXS024); the 'Excellent creative talents support program' of Heilongjiang University of Chinese Medicine (no. 2012RCD19) and the Key Laboratory of Myocardial Ischemia, Harbin Medical University, Chinese Ministry of Education (no. KF201319).

\section{References}

1. Ai J, Wang N, Yang M, Du ZM, Zhang YC and Yang BF: Development of Wistar rat model of insulin resistance. World J Gastroenterol 11: 3675-3679, 2005.

2. Haslam DW and James WPT: Obesity. Lancet 366: 1197-1209, 2005.

3. Langeveld M and Aerts JM: Glycosphingolipids and insulin resistance. Prog Lipid Res 48: 196-205, 2009.

4. Panzer C, Lauer MS, Brieke A, Blackstone E and Hoogwerf B: Association of fasting plasma glucose with heart rate recovery in healthy adults: a population-based study. Diabetes 51: 803-807, 2002.

5. Shepherd PR, Withers DJ and Siddle K: Phosphoinositide 3-kinase: The key switch mechanism in insulin signalling. Biochem J 333: 471-490, 1998.

6. Justinich CJ, Mak N, Pacheco I, et al: The extracellular calcium-sensing receptor (CaSR) on human esophagus and evidence of expression of the CaSR on the esophageal epithelial cell line (HET-1A). Am J Physiol Gastrointest Liver Physiol 294: G120-G129, 2008 .

7. Li GW, Xing WJ, Bai SZ, et al: The calcium-sensing receptor mediates hypoxia-induced proliferation of rat pulmonary artery smooth muscle cells through MEK1/ERK1,2 and PI3K pathways. Basic Clin Pharmacol Toxicol 108: 185-193, 2011.

8. Dvorak MM, Siddiqua A, Ward DT, et al: Physiological changes in extracellular calcium concentration directly control osteoblast function in the absence of calciotropic hormones. Proc Natl Acad Sci USA 101: 5140-5145, 2004.

9. Li HX, Kong FJ, Bai SZ, et al: Involvement of calcium-sensing receptor in oxLDL-induced MMP-2 production in vascular smooth muscle cells via PI3K/Akt pathway. Mol Cell Biochem 362: 115-122, 2012.

10. King AJ: The use of animal models in diabetes research. Br J Pharmacol 166: 877-894, 2012.

11. Vangipurapu J, Stančáková A, Kuulasmaa T, et al: Association between liver insulin resistance and cardiovascular risk factors. J Intern Med 272: 402-408, 2012.

12. El-Serag HB, Tran T and Everhart JE: Diabetes increases the risk of chronic liver disease and hepatocellular carcinoma. Gastroenterology 126: 460-468, 2004.

13. Walker KS, Deak M, Paterson A, Hudson K, Cohen P and Alessi DR: Activation of protein kinase B beta and gamma isoforms by insulin in vivo and by 3-phosphoinositide-dependent protein kinase-1 in vitro: comparison with protein kinase B alpha. Biochem J 331: 299-308, 1998.

14. Leech CA and Habener JF: Regulation of glucagon-like peptide-1 receptor and calcium-sensing receptor signaling by L-histidine. Endocrinology 144: 4851-4858, 2003.

15. Ohkubo E, Aida K, Chen J, et al: A patient with type 2 diabetes mellitus associated with mutations in calcium sensing receptor gene and mitochondrial DNA. Biochem Biophys Res Commun 278: 808-813, 2000.

16. Franke TF, Kaplan DR and Cantley LC: PI3K: downstream AKTion blocks apoptosis. Cell 88: 435-437, 1997.

17. Burgering BM and Coffer PJ: Protein kinase B (c-Akt) in phosphatidylinositol-3-OH kinase signal transduction. Nature 376: 599-602, 1995.

18. Franke TF, Yang SI, Chan TO, et al: The protein kinase encoded by the Akt proto-oncogene is a target of the PDGF-activated phosphatidylinositol 3-kinase. Cell 81: 727-736, 1995.

19. Hajduch E, Litherland GJ and Hundal HS: Protein kinase B (PKB/Akt) - A key regulator of glucose transport? FEBS Lett 492: 199-203, 2001.

20. Diehl JA, Cheng M, Roussel MF and Sherr CJ: Glycogen synthase kinase-3beta regulates cyclin D1 proteolysis and subcellular localization. Genes Dev 12: 3499-3511, 1998.

21. Alessi DR, Andjelkovic M, Caudwell B, et al: Mechanism of activation of protein kinase B by insulin and IGF-1. EMBO J 15: 6541-6551, 1996. 